\title{
Switching emissions of two tetraphenylethene derivatives with solvent vapor, mechanical, and thermal stimuli
}

\author{
SHI JunQing ${ }^{1}$, ZHAO WeiJun ${ }^{1}$, LI CuiHong ${ }^{1}$, LIU ZhengPing ${ }^{1}$, BO ZhiShan ${ }^{1}$, DONG YuPing ${ }^{2}$, \\ DONG YongQiang ${ }^{1 *} \&$ TANG Ben Zhong ${ }^{3,4^{*}}$ \\ ${ }^{1}$ Beijing Key Laboratory of Energy Conversion and Storage Materials, College of Chemistry, Beijing Normal University, Beijing 100875, China; \\ ${ }^{2}$ College of Materials Science and Engineering, Beijing Institute of Technology, Beijing 100081, China; \\ ${ }^{3}$ Department of Chemistry, The Hong Kong University of Science \& Technology (HKUST), Clear Water Bay, Kowloon, Hong Kong, China; \\ ${ }^{4}$ Institute of Biomedical Macromolecules, MOE Key Laboratory of Macromolecular Synthesis and Functionalization, Department of \\ Polymer Science and Engineering, Zhejiang University, Hangzhou 310027, China
}

Received January 30, 2013; accepted March 7, 2013; published online June 6, 2013

\begin{abstract}
Two derivatives of tetraphenylethene (TPE) were synthesized through one step cross McMurry coupling reaction. Both luminogens exhibit aggregation-induced emission (AIE) and crystallization induced emission enhancement (CIEE). The emissions of both luminogens could be switched between blue and green through reversible modulation of morphology with thermal, organic solvent fuming and mechanical stimuli. Thus we provide a possible design strategy for emission switching materials.
\end{abstract}

aggregation-induced emission (AIE), crystallization-induced emission enhancement (CIEE), mechanochromic fluorescence

Citation: Shi J Q, Zhao W J, Li C H, et al. Switching emissions of two tetraphenylethene derivatives with solvent vapor, mechanical, and thermal stimuli. Chin Sci Bull, 2013, 58: 2723-2727, doi: 10.1007/s11434-013-5868-1

Stimulus responsive luminescent materials have drawn many attentions due to their fundamental importance and potential application in sensors [1,2], optical recording, and security ink [3]. Of particular interest are those materials whose emission could be switched between different colors or between varied brightness in the solid state [4]. Though molecular structure modification is the most common approach for modulating emissions, limited examples exhibiting efficient and reversible fluorescence switching in the solid state have been achieved because of the insufficient conversion and irreversible reactions involved in most chemical reactions in the solid state [5].

Some materials exhibit morphology dependent luminescence, thus, their emissions could be switched among different colors through reversibly modulating molecular packing patterns in the solid state instead of changing chemical structures [6-9]. However, there is still no clear design strategy for such materials though several examples

*Corresponding authors (email: dongyq@bnu.edu.cn; tangbenz@ust.hk) have been reported.

Efficient solid luminescent materials are preferred because luminogens are normally used in the solid state in most applications. However, a notorious problem associated with the emissions of most luminogenic materials in the solid state is aggregation-caused quenching (ACQ): during aggregate formation, the luminogen molecules are located closely, facilitating the formation of detrimental species such as excimers [10]. Closely related to the ACQ phenomenon is the crystallization of dye molecules, which normally red-shifts fluorescence of the luminogens and weakens their emissions. Many efforts have been devoted to fabricate amorphous films with high glass transition temperature to avoid crystallization of luminogens.

We have observed a phenomenon exactly opposite to the ACQ effect: a series of propeller-shaped molecules that are nearly nonemissive in solution are induced to emit efficiently when forming aggregates. Thus the phenomenon is coined as aggregation-induced emission (AIE) [11]. During our study on the AIE phenomenon, we have noted that 
many AIE-active dyes emit bluer lights in higher efficiencies in the crystalline phase than in their amorphous phase $[10,12,13]$. The crystallization-induced emission enhancement (CIEE) effect enables readily switching the fluorescence of luminogens between various states through reversibly modulating the packing patterns of luminogens with external stimuli such as fuming, thermal, and mechanical stimuli $[6,14,15]$.

Some AIE active materials have already been reported to exhibit emission color switching, however, most of them were synthesized through multiple steps $[6,16]$. In this letter, we reported two AIE and CIEE active luminogens synthesized through one step reaction. Emissions of both luminogens could be switched between blue and green through reversibly transforming their morphology between crystalline and amorphous states with solvent fuming, heating, and mechanical stress.

Biphenyl-4-yltriphenylethene (1) and di(biphenyl-4-yl) diphenylethene (2) were synthesized through cross McMurry coupling reaction of benzophenone and 4-phenylbenzophenone, and their structures are shown in Scheme 1. McMurry coupling reaction can only produce E/Z mixtures of luminogen $\mathbf{2}$, which are difficult to separate through normal purifying method, thus the $E / Z$ mixtures of $\mathbf{2}$ are studied as a whole. Detailed synthetic route and characterizations of the products are described in the Supporting Information (Figure S1).

Similar to other TPE derivatives, $\mathbf{1}$ also exhibits AIE activity [17]. The solution of $\mathbf{1}$ is nearly nonemissive, while suspension of $\mathbf{1}$ formed in the mixture of acetonitrile and water is highly emissive (Figure S2). Luminogen $\mathbf{2}$ has already been reported to be AIE active [18].

In addition to the suspension of luminogens, photophysical properties of luminogens in solid stated are more attractive as many luminogens are used in the solid state in real application. Green emissive amorphous solids of both luminogens were prepared through quenching of their melt (Figure 1(b) and (g)). The emissions of amorphous solids of 1 and 2 peaked at 495 and $505 \mathrm{~nm}$ with $12.7 \%$ and $10.2 \%$ quantum yield $\left(\Phi_{\mathrm{f}}\right)$ respectively (Figure $1(\mathrm{e})$ and $(\mathrm{j})$ ). Crystals of 1 (Figure 1(a)) and 2 (Figure 1(f)), obtained through slow evaporation of their solutions, emit blue lights peaked at 455 and $444 \mathrm{~nm}$ respectively with $34.45 \%$ and $28.58 \%$ quantum yield. It is clear that crystals of both luminogens exhibit bluer emission in nearly three times higher efficiencies than their amorphous solids, that is, both luminogens are CIEE active. Similar to TPE, molecules of both luminogens take twisted conformations due to the intramolecular steric hindrance (Figure S3). Thus the propeller-like conformations rule out specific strong intermolecular interactions (such as $\pi-\pi$ stacking or $\mathrm{H} / \mathrm{J}$-aggregates), which may quench the emission of traditional pane-like luminogens. Molecules in crystal pack in tighter patterns than those in amorphous solid, and the molecular conformations in the luminogen crystals are locked/stabilized by multiple $\mathrm{C}-\mathrm{H} \cdots$ $\pi$ bonds (Figure S3). Thus the structural rigidification makes the crystals stronger emitters. The blue-shifted PL spectra may be due to the more twisted conformation of molecules in crystals.

Though we have reported several TPE derivatives exhibiting morphology dependent emission, luminogens $\mathbf{1}$ and $\mathbf{2}$ show more distinct contrasts in emission color between amorphous and crystalline states (40 and $60 \mathrm{~nm}$ blue-shift compared with their amorphous cousins). Molecules of $\mathbf{1}$ and $\mathbf{2}$ own one and two more phenyl rings respectively than tetra-alkoxy substituted TPE [19], which may afford the larger difference in conjugation degree and hence the sharp contrast in emission color between amorphous and crystalline states of luminogens.

The CIEE effect of $\mathbf{1}$ and $\mathbf{2}$ prompts us to explore the possibility of reversibly modulating their emissions through a simple engineering process. Amorphous solids of luminogens normally transform to crystalline phase upon heating. The emissions of amorphous solid of $\mathbf{1}$ and $\mathbf{2}$ transferred from green to blue when annealed at 95 and $150^{\circ} \mathrm{C}$ for 7 min respectively (Figure $1(\mathrm{c})$ and (h)). In addition to visual inspection, the PL spectra of annealed samples fit well with their pristine crystals, indicating reverting back to pristine crystals upon heating (Figure 1(e) and (j)). The powder X-ray diffraction (XRD) curves of annealed samples also coincide with their crystalline samples, further verifying the transformation from amorphous to crystalline states (Figure S4). The amorphous solid could be obtained again through quenching of luminogens melts. Thus the emissions of both luminogens $\mathbf{1}$ and $\mathbf{2}$ could be switched between blue and green through a pure thermal method. Heat-mode phase-change technology has been used in the rewritable optical media. A luminescence-based process is

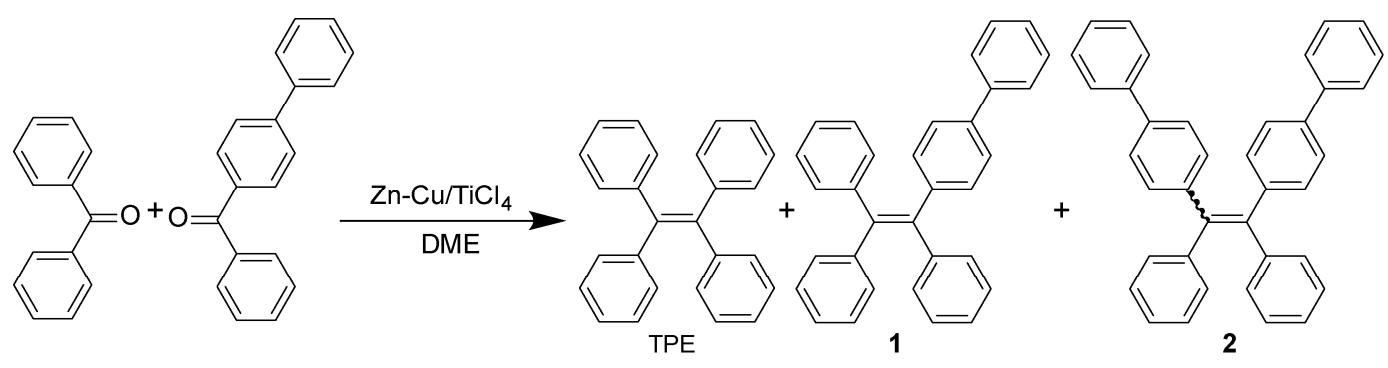

Scheme 1 The synthetic route of compounds $\mathbf{1}$ and $\mathbf{2}$. 


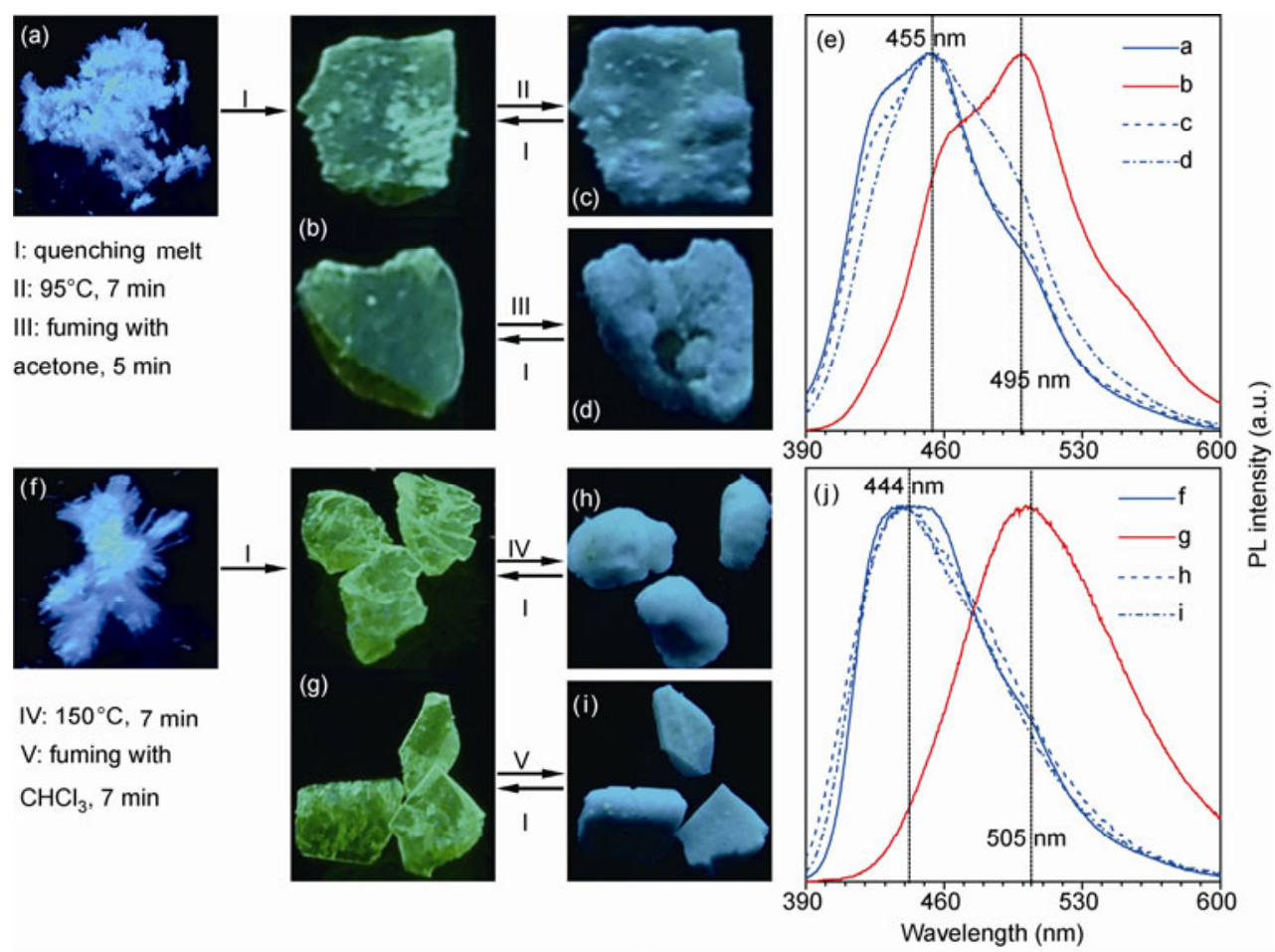

Figure 1 Images of 1 (a)-(d) and 2 (f)-(i): (a), (f) pristine crystal; (b), (g) amorphous solids; (c), (h) thermal treated and (d), (i) solvent fumed amorphous solids; PL spectra of samples of $\mathbf{1}$ (e) and $\mathbf{2}$ (j) in the images. Excitation wavelength: $350 \mathrm{~nm}$. Photographs were taken under UV illumination.

of advantage due to its higher sensitivity, lower background noises. Luminogens $\mathbf{1}$ and $\mathbf{2}$ are thus promising candidates for innovative applications in optical information storage systems.

Both 1 and $\mathbf{2}$ exhibit vapochromic fluorescence. The green emissive amorphous solid of luminogen 1 transformed to blue emissive crystals upon fuming with acetone for 5 min (Figure 1(b) and (d)). Amorphous solid of 2 also crystallized when fumed with chloroform for $7 \mathrm{~min}$ with emission changing from green to blue (Figure $1(\mathrm{~g})$ and (i)) though it contains both $E$ and $Z$ stereoisomers, indicating that the presence of isomers does not affect the transformation between crystalline and amorphous phase. Thus the emissions of luminogens $\mathbf{1}$ and $\mathbf{2}$ can also be switched between blue and green through repeating fuming and heating, which enables their potential application as chemosensor for detecting volatile organic compounds.

Several AIE active luminogens have been reported to exhibit mechanochromic luminescence. Thus we check the mechanical response of luminescence of $\mathbf{1}$ and 2. Emission of crystals of $\mathbf{2}$ changed from blue to green upon grinding in a mortar (Figure 2(a) and (b)). After annealing, emission of the ground powder reverted back to that of the original crystal (Figure 2(c)). The PL spectrum of the ground solid of 2 fits well with that of amorphous sample, while after annealing, the PL of the ground solid overlapped with that of pristine crystals of 2 (Figure 2(e)). Similar to 2, luminogen 1 also exhibits mechanochromic fluorescence (Figure S5). Thus emissions of both luminogens could be modulat- ed reversibly between blue and green through repeatedly grinding and heating processes (Figures S6 and S7).

To disclose the mechanism of mechanochromic fluorescence of $\mathbf{1}$ and 2, powder X-ray diffraction (XRD) was carried out on the pristine blue crystals, ground and annealed powder of both luminogens (Figure S8). The powder XRD curves of both original blue crystals and annealed samples display many sharp and intense reflection peaks and coincide with each other, indicating their same crystalline orders. Diffraction curve of ground powders also exhibits some reflections, which agree with but not as many and sharp as those of their original crystals, hinting its partly amorphous essence. Hence the mechanochromic fluorescences of $\mathbf{1}$ and $\mathbf{2}$ are caused by the amorphization upon grinding.

Fuming can also facilitate crystallization of ground solid of both luminogens, thus, the emissions of $\mathbf{1}$ and $\mathbf{2}$ could also be tuned reversibly through repeatedly grinding and fuming (Figures S9 and S10).

The mechanochromic fluorescence of both luminogens prompts us to study their possibility as an optical recording material. Luminogen $\mathbf{2}$ was ground on a piece of weighing paper, and a green emissive paper was obtained (Figure 3). Then emission of the paper turned from green to blue upon heating, in accordance with the transition from amorphous to crystalline phase. Then we wrote "BNU" on the paper, and green letters appeared on the blue background due to the amorphization of $\mathbf{2}$ in the written area. The green letters was erased upon heating due to crystallization of $\mathbf{2}$ in the letters area. The writing and erasing process can be repeated 

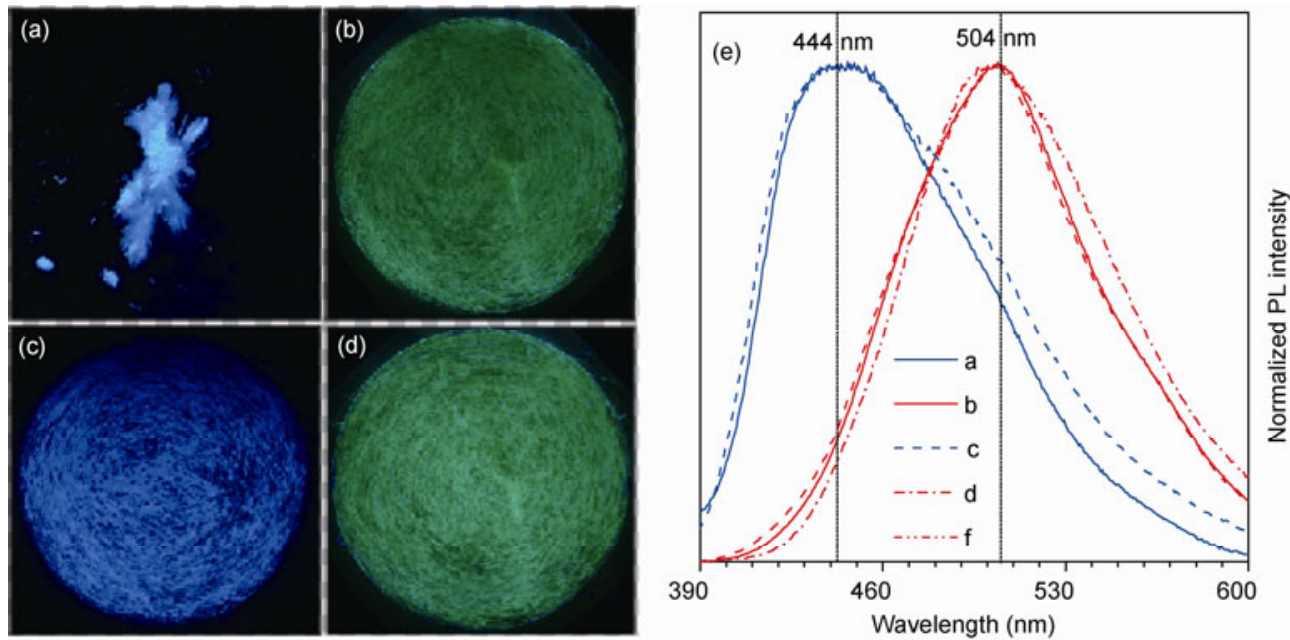

Figure 2 Images of 2: (a) crystals; ground solid (b) before, (c) after annealing, and (d) regrinding. (e) PL spectra of samples in the images. PL spectrum of (line f) amorphous solid of $\mathbf{2}$ is also listed for comparison. Annealing details: $175^{\circ} \mathrm{C}, 15 \mathrm{~min}$. Excitation wavelength: $350 \mathrm{~nm}$. Photographs were taken under UV illumination.
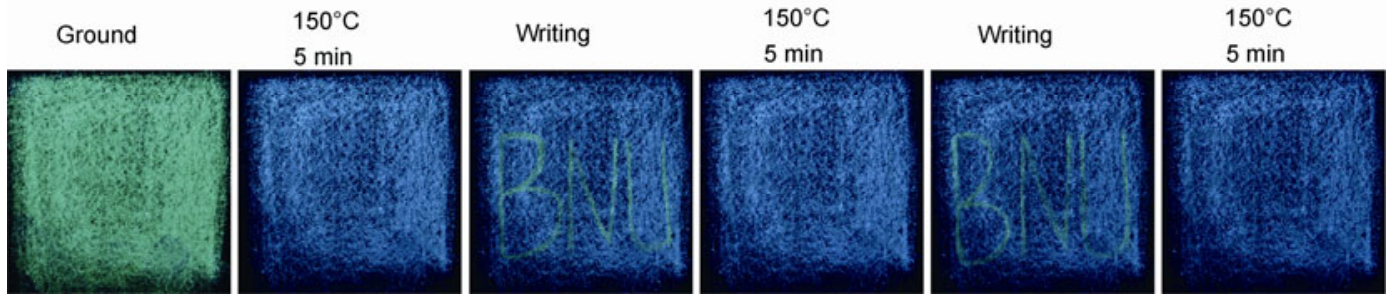

Figure 3 Procedures of repeating writing and erasing process using luminogen 2 as the emissive materials. Images were taken on a weighing paper under UV irradiation at $365 \mathrm{~nm}$.

many times through repeating writing and heating processes.

In conclusion, the emissions of both luminogens could be tuned between blue and green in the solid state through modulating the morphology of luminogens between crystalline and amorphous states with thermal, fuming and mechanical stimuli. Many AIE and CIEE active luminogens exhibit high efficient emission in solid state. The propeller-like conformations of those materials afford loose molecular packing patterns and morphology dependent emissions, thus facilitate reversible modulation of their morphology to switch their emissions. Hence we provide a possible design strategy of luminescent materials exhibiting emission switching in solid states.

This work was partially supported by the National Natural Science Foundation of China (51173018), National Basic Research Program of China (2013CB834704, 2011CB935702), the Fundamental Research Funds for the Central Universities, RPC Grants of HKUST (RPC11SC09), and the Research Grants Council of Hong Kong (HKUST2/CRF/10).

1 Zhu W, Huang X M, Guo Z Q, et al. Chem Commun, 2012, 48: 1784-1786

2 Xue W X, Zhang G X, Zhang D Q, et al. Org Lett, 2010, 12:
2274-2277

3 Kishimura A, Yamashita T, Yamaguchi K, et al. Nat Mater, 2005, 4: 546-549

4 Mutai T, Satou H, Araki K. Nat Mater, 2005, 4: 685-687

5 Zhao Y F, Gao H Z, Fan Y, et al. Adv Mater, 2009, 21: 3165-3169

6 Chi Z, Zhang X, Xu B, et al. Chem Soc Rev, 2012, 41: 3878- 3896

7 Teng M J, Jia X R, Yang S, et al. Adv Mater, 2012, 24: 1255-1261

8 Sagara $Y$, Kato T. Nat Chem, 2009, 1: 605-610

9 Abe Y, Karasawa S, Koga N. Chemistry-Europ J, 2012, 18: $15038-15048$

10 Hong Y N, Lam J W Y, Tang B Z. Chem Soc Rev, 2011, 40: 5361-5388

11 Luo J D, Xie Z L, Lam J W Y, et al. Chem Commun, 2001, $1740-1741$

12 Dong Y Q, Lam J W Y, Qin A J, et al. Chem Commun, 2007, 40-42

13 Huang J, Sun N, Yang J, et al. J Mater Chem, 2012, 22: 12001-12007

14 Luo X L, Li J N, Li C H, et al. Adv Mater, 2011, 23: 3261-3265

15 Gu X, Yao J, Zhang G, et al. Adv Funct Mater, 2012, 22: 4862-4872

16 Xu B J, Chi Z G, Zhang J Y, et al. Chem-Asian J, 2011, 6: $1470-1478$

17 Huang J, Yang X, Wang J, et al. J Mater Chem, 2012, 22: 24782484

18 Dong Y Q, Lam J W Y, Qin A J, et al. Appl Phys Lett, 2007, 91: 011111-011113

19 Luo X, Zhao W, Shi J, et al. J Phys Chem C, 2012, 116: $21967-21972$

Open Access This article is distributed under the terms of the Creative Commons Attribution License which permits any use, distribution, and reproduction in any medium, provided the original author(s) and source are credited. 


\section{Supporting Information}

Figure S1 UV spectra of 1 and 2 in acetonitrile. Concentration: $10 \mu \mathrm{mol} / \mathrm{L}$.

Figure S2 (a) PL spectra of $\mathbf{1}$ in acetonitrile/water mixtures with different water fractions $\left(f_{\mathrm{w}}\right)$. (b) Plots of its PL peak intensity vs. water content of the solvent mixture. Inset: photographs of $\mathbf{1}$ in the acetonitrile/water mixtures with $0 \%$ and $90 \%$ water fraction were taken under UV illumination. Dye concentration: $10 \mu \mathrm{mol} / \mathrm{L}$; excitation wavelength: $350 \mathrm{~nm}$.

Figure S3 View of molecule conformation and weak interaction in crystal of 2.

Figure S4 Powder XRD patterns of 1(a) and 2(b): Pristine crystal, amorphous solid before and after annealed.

Figure S5 Images of 1: (a) crystals; ground solid (b) before, (c) after annealing, and (d) regrinding. (e) PL spectra of samples in the images. PL spectrum of (line f) amorphous solid of $\mathbf{1}$ is also listed for comparison. Annealing details: $100^{\circ} \mathrm{C}$ for 7 min. Excitation wavelength: $350 \mathrm{~nm}$. Photographs were taken under UV illumination.

Figure S6 (a) Normalized PL spectra and (b) photos of $\mathbf{1}$ in three repeating cycles of grinding (red lines in (a)) and annealing (blue lines in (a)) (100 ${ }^{\circ} \mathrm{C}$ for $7 \mathrm{~min}$ ).

Figure S7 (a) Normalized PL spectra and (b) photos of 2 in three repeating cycles of grinding (red lines in (a)) and annealing (blue lines in (a)) (175 ${ }^{\circ} \mathrm{C}$ for $15 \mathrm{~min})$.

Figure S8 Powder XRD patterns of 1(a) and 2(b): Pristine crystal, ground solid before and after annealed.

Figure S9 (a) Normalized PL spectra and (b) photos of $\mathbf{1}$ in three repeating cycles of grinding (red lines in (a)) and fuming (blue lines in (a)). Fuming detail: Acetone for $9 \mathrm{~min}$.

Figure S10 (a) Normalized PL spectra and (b) photos of 2 in three repeating cycles of grinding (red lines in (a)) and fuming (blue lines in (a)). Fuming detail: Chloroform for $15 \mathrm{~min}$.

The supporting information is available online at csb.scichina.com and www.springerlink.com. The supporting materials are published as submitted, without typesetting or editing. The responsibility for scientific accuracy and content remains entirely with the authors. 